\title{
Overview of the Current Status of IFMIF-DONES Test Cell Biological Shielding Design
}

Kuo Tiana ${ }^{*}$, Begoña Ahedo ${ }^{b}$, Frederik Arbeiter ${ }^{a}$, German Barrera ${ }^{b}$, Łukasz Ciupińskic, Tamás Dézsid, Jonathan Horne ${ }^{e}$, Dániel Kovács ${ }^{\mathrm{d}}$, Joaquin Molla ${ }^{\mathrm{b}}$, Fernando Mota ${ }^{\mathrm{b}}$, Yuefeng Qiuª, Florian Schwabª ${ }^{\mathrm{a}}$ Marcin Siwek ${ }^{\mathrm{c}}$, Mátyás Tóth ${ }^{\dagger}$, Tamás Vargag ${ }^{9}$, Angel Ibarrab

aKIT, Germany, ${ }^{b}$ CIEMAT, Spain, "Warsaw University of Technology, Poland, dWigner RCP / C3D Ltd., Hungary

${ }^{e}$ RACE, Culham Science Centre, United Kingdom , ${ }^{f} H A S$, Wigner RCP, Hungary, gWigner RCP / Fuziotech Ltd., Hungary

\section{Top Shielding Plugs (TSPs)}

$\square$ TSPs including USP \& LSP $\square$ Geometry adapted for RH

LSP actively cooled by helium due to high nuclear heating

$\square$ Reinforcement and Cooling pipes arranged in LSP

$\square$ Cooling capacity being approved
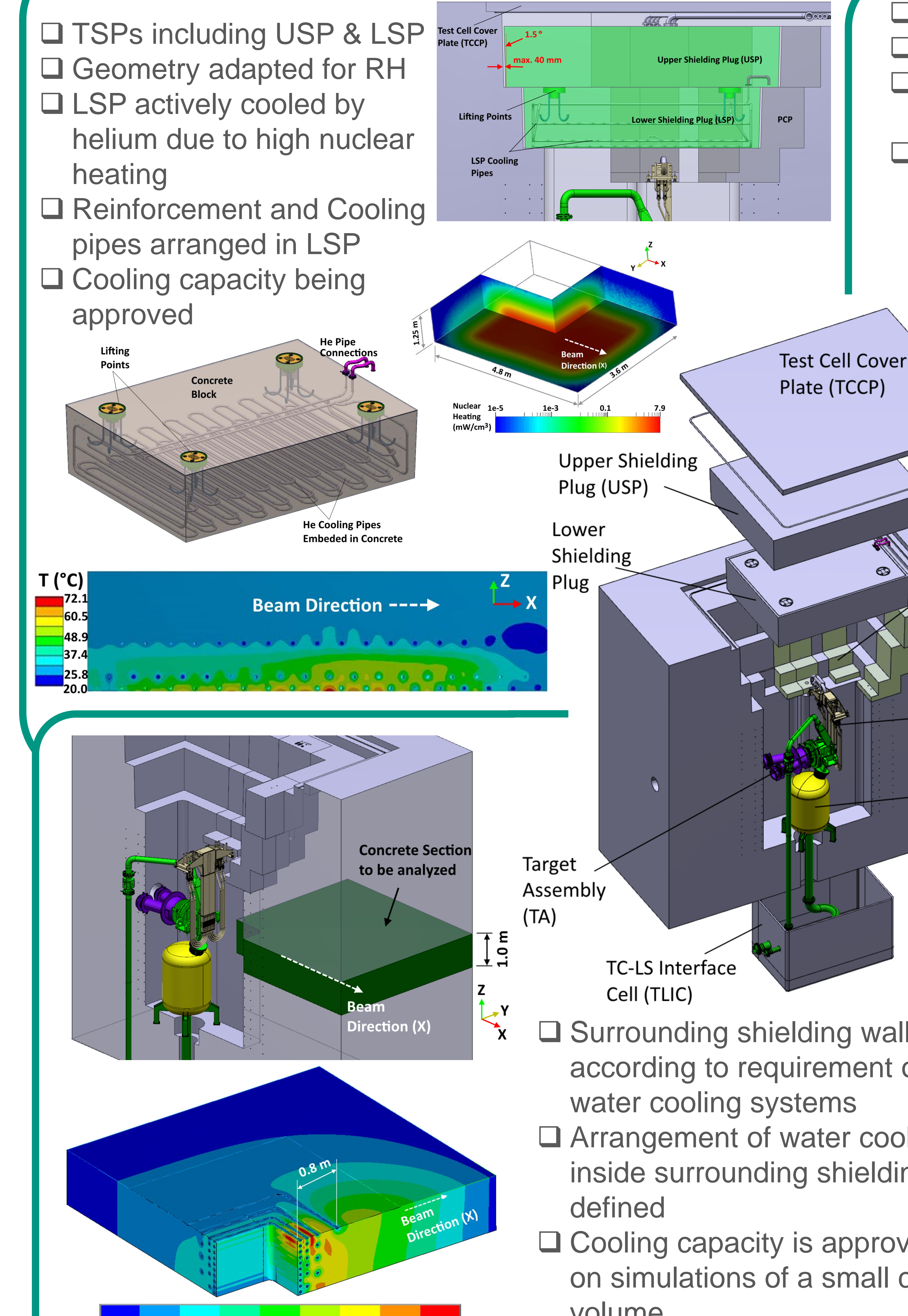

Pipe Arrangement and Temperature Map During Irradiation Experiments Upper Shielding
Plug (USP)
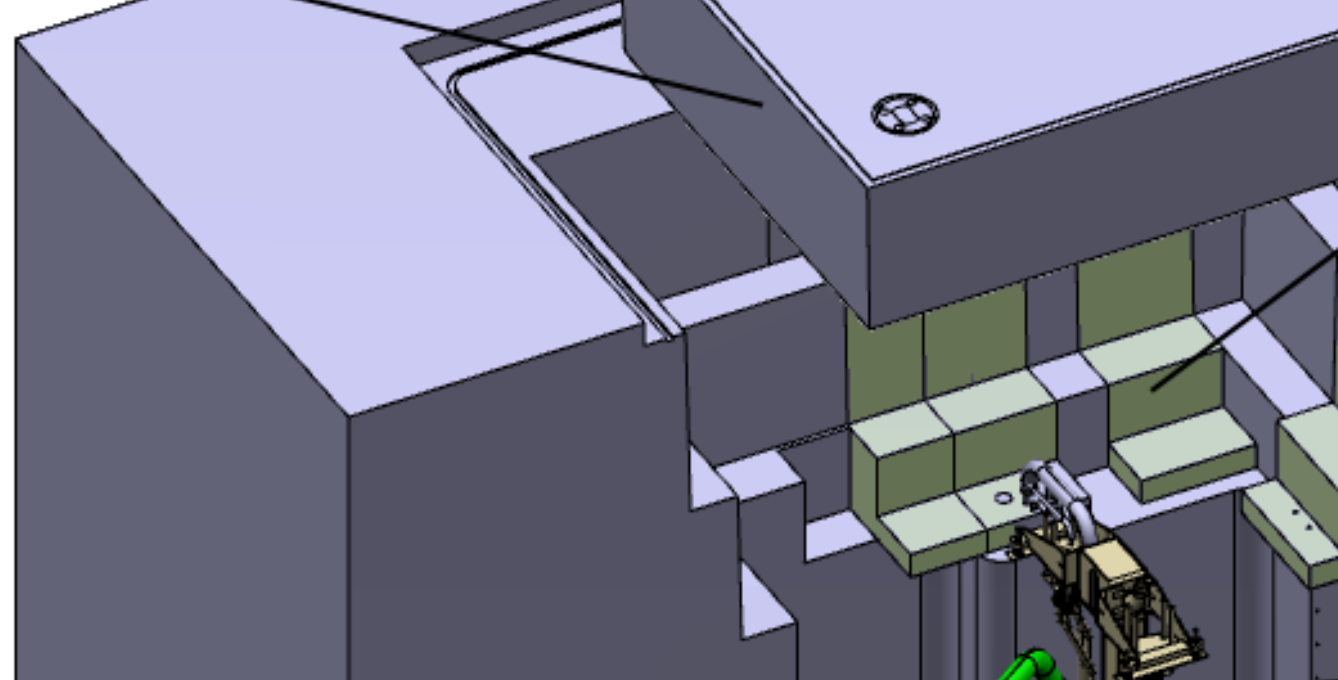

Assembly

(TA)

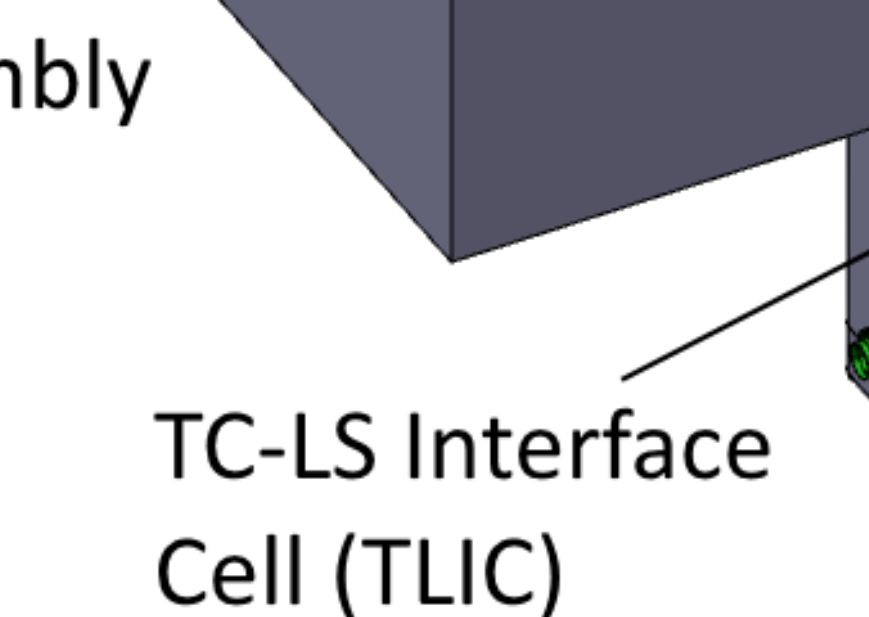
water cooling systems defined volume implemented $\begin{array}{llllllllll}22.1 & 29.0 & 35.9 & 42.7 & 49.6 & 56.5 & 63.4 & 70.2 & 77.1 & 84.0^{\circ} \mathrm{C}\end{array}$

\section{Piping and Cabling Plugs (PCPs)}

$\checkmark$ PCPs accommodates all pipe/cable penetrations

$\square$ PCP design based on IFMIF-EVEDA design

$\square$ Lower end of PCP is extended for convenient connection arrangement

$\square$ Embedded pipes have several bends to minimize neutron streaming

\section{$\square$ Insulation materials} applied to helium pipes

$\square$ Additional active cooling not required

\section{$\square$ Further update on} geometry required
Sealing Gasket Plate (TCCP)

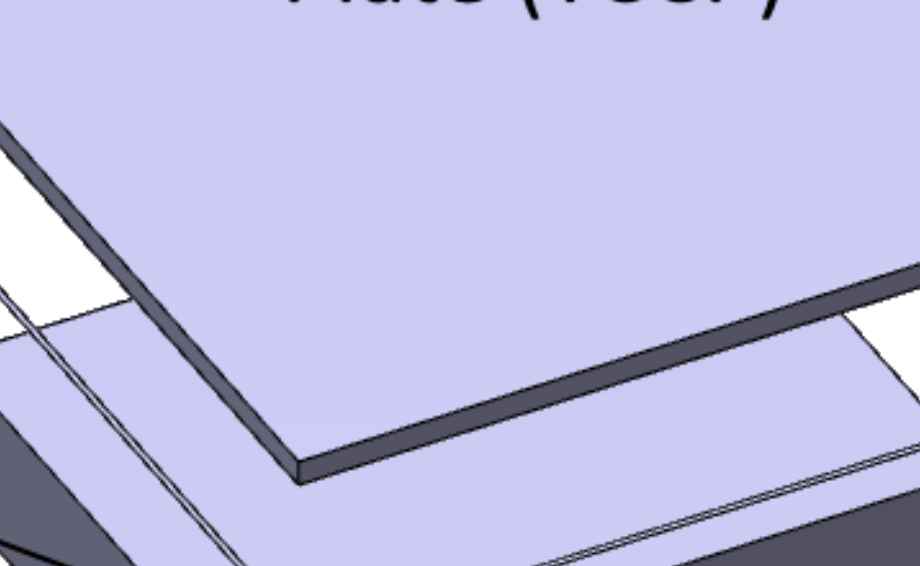
Lower Shielding

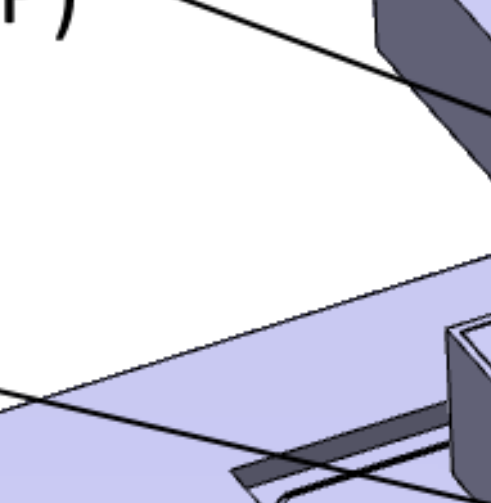

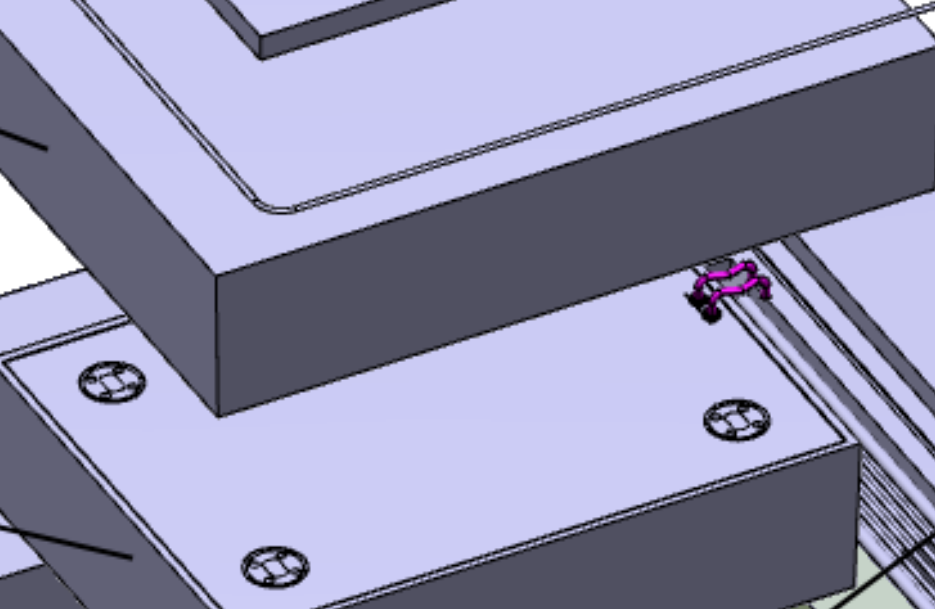

Piping and

$\square$ Detailed

internal

Cabling Plugs structure to

Cablin
(PCPs)

be completed
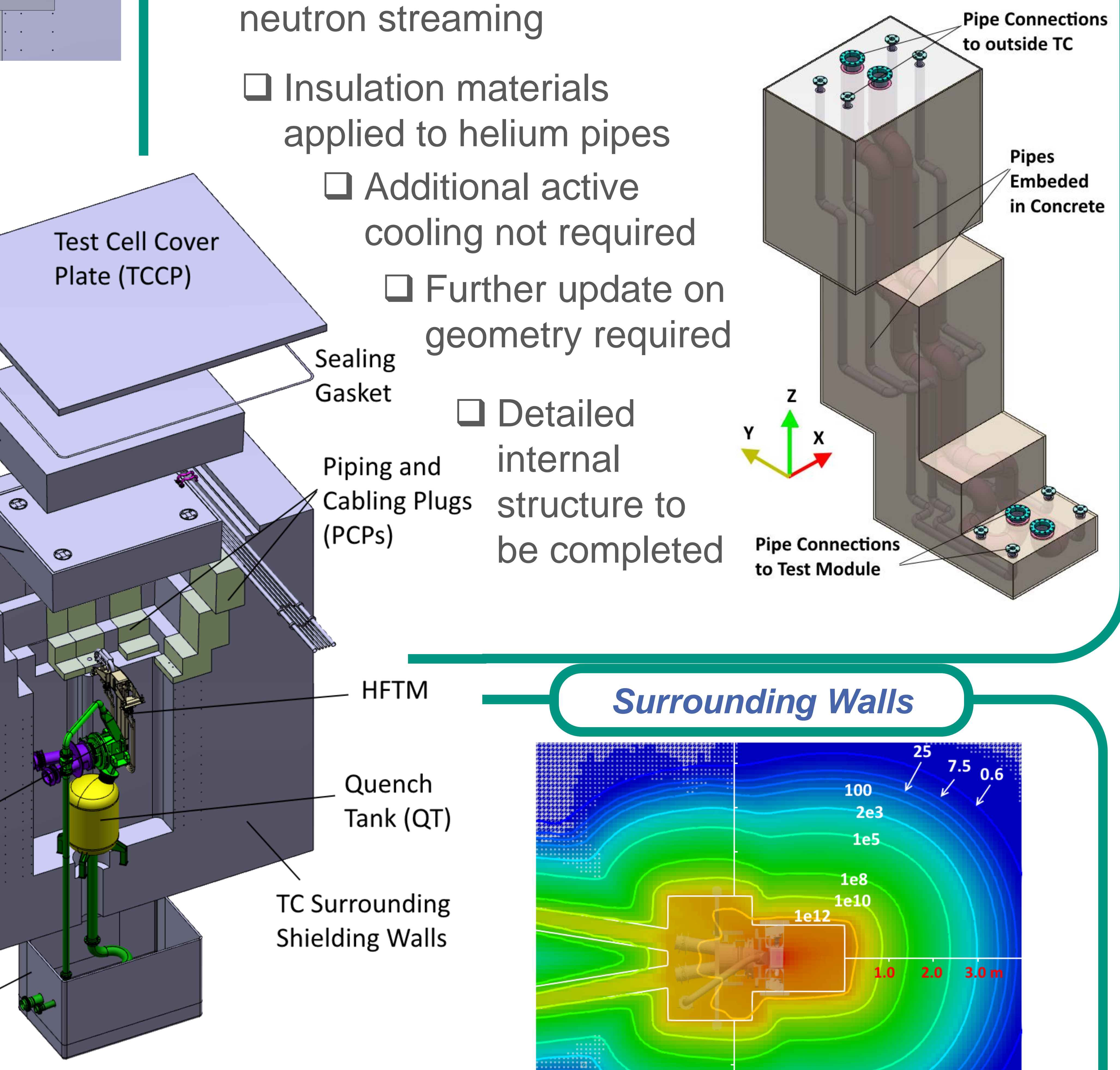

$\square$ Surrounding shielding walls updated according to requirement of $\mathrm{RH}$ and

$\checkmark$ Arrangement of water cooling pipes inside surrounding shielding walls

$\square$ Cooling capacity is approved based on simulations of a small controlled

Further analysis on $1 / 4$ TC will be

*Corresponding author: kuo.tian@kit.edu
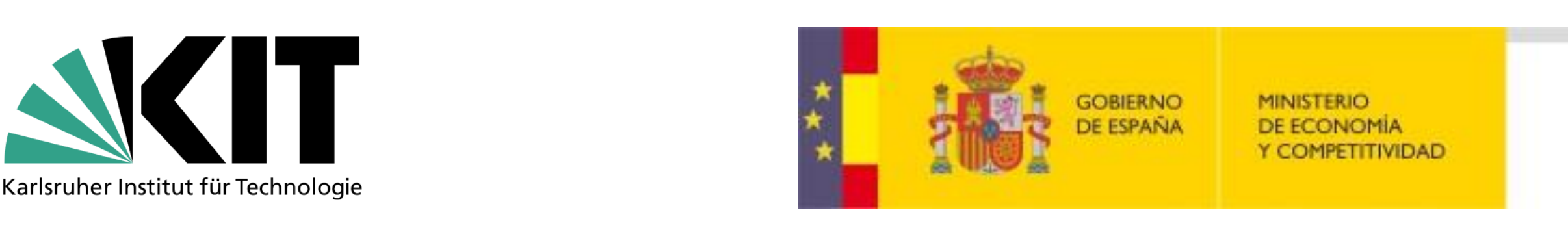

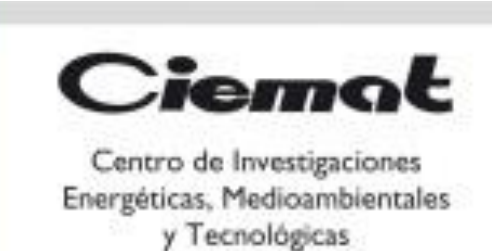

Politechnika

IVarszawska

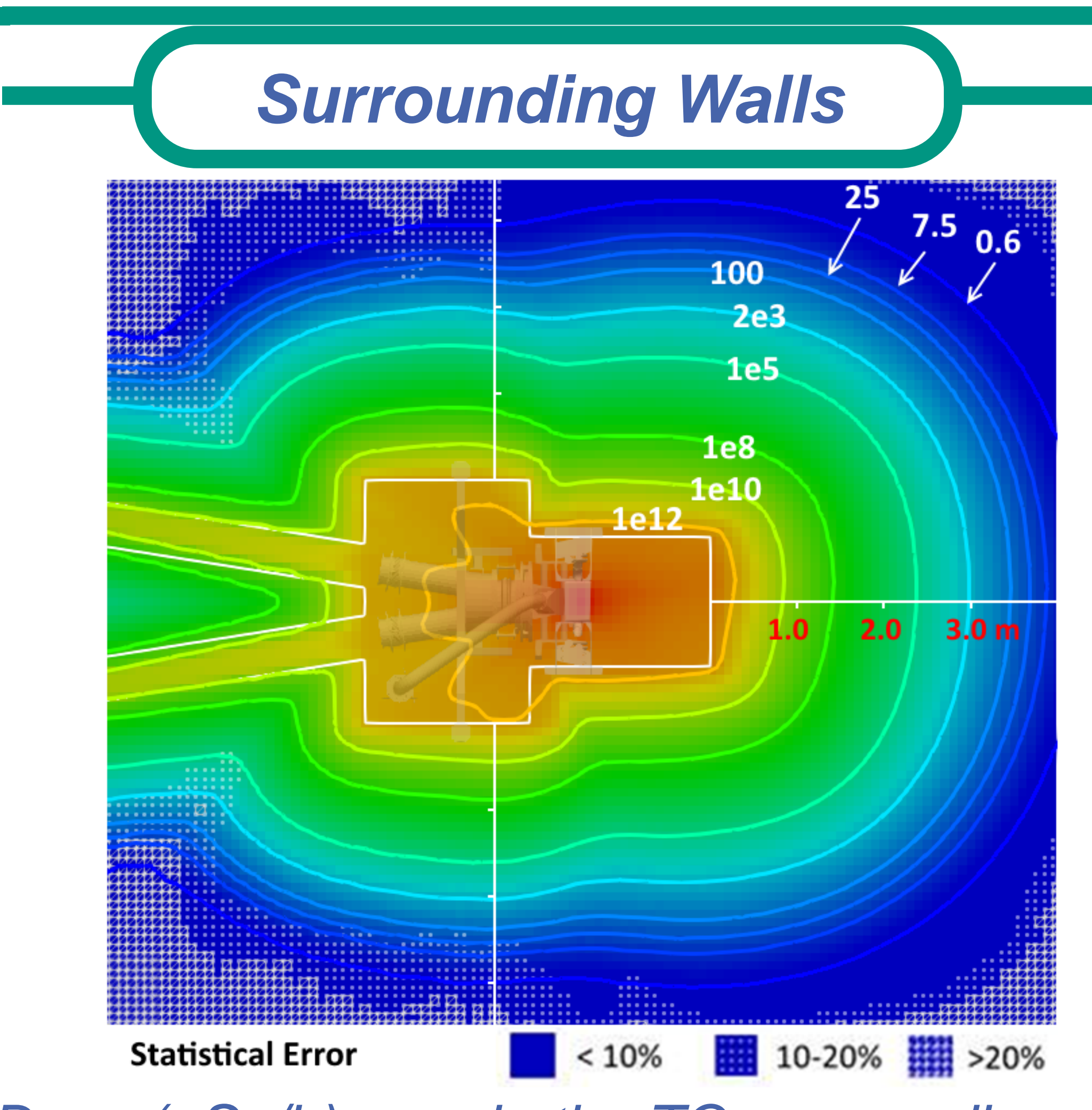

Dose ( $\mu \mathrm{Sv} / \mathrm{h})$ map in the TC surrounding wall

\section{SUMMARY}

$\square$ Design of DONES-TC biological shielding components updated

口TC surrounding shielding walls: geometry updated, active cooling pipes preliminarily arranged

$\square$ PCPs: geometry updated, piping and cabling defined

- Lower Shielding Plug: detailed structure and calculations implemented 